\title{
Double-sided Thomson coil based actuator: finite element design and performance analysis
}

DOI:

10.1049/cp.2016.0201

\section{Document Version}

Accepted author manuscript

Link to publication record in Manchester Research Explorer

\section{Citation for published version (APA):}

Barnes, M., Shuttleworth, R., \& Vilchis-Rodriguez, D. (2016). Double-sided Thomson coil based actuator: finite element design and performance analysis. In IET Power Electronics, Machines and Drives Conf, April 2016, Glasgow https://doi.org/10.1049/cp.2016.0201

\section{Published in:}

IET Power Electronics, Machines and Drives Conf, April 2016, Glasgow

\section{Citing this paper}

Please note that where the full-text provided on Manchester Research Explorer is the Author Accepted Manuscript or Proof version this may differ from the final Published version. If citing, it is advised that you check and use the publisher's definitive version.

\section{General rights}

Copyright and moral rights for the publications made accessible in the Research Explorer are retained by the authors and/or other copyright owners and it is a condition of accessing publications that users recognise and abide by the legal requirements associated with these rights.

\section{Takedown policy}

If you believe that this document breaches copyright please refer to the University of Manchester's Takedown Procedures [http://man.ac.uk/04Y6Bo] or contact uml.scholarlycommunications@manchester.ac.uk providing relevant details, so we can investigate your claim.

\section{OPEN ACCESS}


This paper is a postprint of a paper submitted to and accepted for publication by, and is subject to Institution of Engineering and Technology Copyright. The copy of record is available at IET Digital Library

\title{
Double-sided Thomson coil based actuator: finite element design and performance analysis
}

\author{
D.S. Vilchis-Rodriguez*, R. Shuttleworth*, M. Barnes* \\ * The University of Manchester, School of Electrical and Electronic Engineering, Power Conversion Group. \\ Sackville Street Building, Manchester, UK, M13 MPL \\ Damian.Vilchis-rodriguez@manchester.ac.uk
}

Keywords: DC breaker, ultra-fast actuator, double sided Thomson coil, linear driver, energy conversion efficiency.

\begin{abstract}
In this paper the electrical to mechanical energy conversion efficiency of a double-sided Thomson coil actuator is investigated by means of finite element simulations. It is shown that performance gains compared with the conventional Thomson coil actuator are possible and in line with theoretical analysis. Based on a sensitivity assessment, fine tuning of a double-sided Thomson coil actuator has been conducted, which predicts energy conversion efficiencies as high as $34 \%$. This efficiency considerably surpasses efficiencies reported in research literature for other magnetic repulsion based actuators. It is also shown that the doublesided Thomson coil actuator exceeds substantially the mechanical performance of a single-sided Thomson coil design.
\end{abstract}

\section{Introduction}

In order to better integrate renewable power generation into traditional AC power systems the implementation of multiterminal HVDC networks has been proposed [1, 2, 3]. Due to their flexibility, Voltage Source Converter (VSC) based HVDC systems are seen as the most suitable alternative. VSC based HVDC systems allow independent control of active and reactive power, and bidirectional power flow by reversing current direction. However the high power IGBTs present in VSC HVDC stations possess poor overcurrent capability [4]. Consequently during a DC link fault very fast acting protection and fault disconnection is essential to ensure safe operation $[5,6]$.

At present only solid-state interrupters are able to brake fault current in one millisecond or less [5, 7]. However the relatively high conduction losses of solid-state breakers reduce their practicality $[6,8]$. On the other hand mechanical breakers have low conduction loss, but rely on a relatively slow mechanically operated switch to provide voltage isolation. In order to improve the performance of breakers a combination of solid-state and mechanically operated switches has been proposed to achieve HVDC current interruption $[5,6]$, which use ultra-fast actuators to operate the mechanical switch. These are known as hybrid breakers. For ultra-fast actuation the preferred actuator technology is based on the Thomson coil (TC) design $[6,9,10]$. In this actuator the magnetic field from a flat spiral coil, with a low number of turns, interacts with a conductive plate or armature, Fig. 1. A current impulse injected in the coil results in a time varying magnetic field that produces eddy currents in the armature. Due to the direction of the armature induced currents, a repulsive magnetic force develops between armature and coil. Using this principle opening speeds in excess of $20 \mathrm{~m} / \mathrm{s}$ have been reported [9-13]. The main drawback of this actuation mechanism is the low efficiency, typically in the 5-14\% range, which requires a high energy source to be readily available. The low efficiency of the TC actuator is attributed to the decreasing magnetic link between coil and armature as the gap increases [11, 13].

In order to minimize the energy requirements of a TC based interrupter, mass and stroke length minimization have been attempted. Thus a high number of lightweight, short-stroke and electrically series-connected switches have been proposed for medium voltage breakers $[9,10]$. However for very high voltages and fault currents typical of HVDC systems this practice is not suitable: moving mass and stroke length are governed by the carrying current and voltage levels respectively, and a large array of series and parallel connected mechanical switches would be necessary. The development of a more efficient actuator that allows high mass displacement at long strokes is required. In this regard a repulsion driven design comprised of two opposing coils with a higher efficiency $(23 \%)$ than the TC actuator, and which does not rely on eddy currents, was proposed in $[11,13]$. However the extreme acceleration of the moving element and the effect this has on the flexible electric connections raises reliability concerns. A high efficiency solution which does not involve a moving coil is preferable.

Due to its working principle the TC actuator is a unidirectional device and a second opposing coil is necessary to provide bidirectional displacement. When a coil is excited only the magnetic flux lines on one side of the excited coil interact with the armature. Thus one half of the generated magnetic flux is unused. A second armature placed on the other side of the coil may thus be used to improve magnetic flux utilisation and these double-sided TC designs have been proposed for use in high speed switches [14]. However their efficiency and overall suitability for long stroke, ultra-high speed applications has not been fully assessed. 


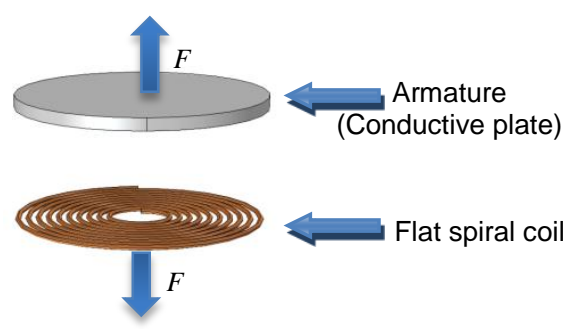

Fig. 1 TC actuator schematic diagram.

In this work the use of a double-sided Thomson coil (DS-TC) actuator is proposed in order to make better use of the coil produced magnetic flux, thus increasing efficiency over the conventional single-sided TC design. Its capability for high efficiency operation at long strokes has been investigated and compared with a conventional TC design. A sensitivity analysis was conducted in order to better understand the factors that affect DS-TC performance. A high efficiency, high-performance DS-TC is realized, and has been demonstrated to exhibit a higher efficiency than a single-sided TC design can achieve.

\section{Double sided Thomson coil actuator}

The asymmetric nature of a TC design ensures that the magnetic flux produced by the coil is not fully exploited. Thus there may be performance gains if a symmetrical arrangement is used. In order to investigate this, a numerical model of the single-sided TC was initially built using FEA software to analyse performance. FEA software has been shown before to predict accurately the behaviour of magnetic repulsion based linear drivers [11, 13]. The numerical simulations were performed using COMSOL 5.1 and involved the simultaneous solution of solid mechanics, electrical circuits and electromagnetic equations over a simulation time of $2 \mathrm{~ms}$, which is comparable with reported hybrid HVDC breaker operation times [6]. For simplicity 2D axial symmetry and a copper armature were assumed in all cases. The coil was excited by a capacitor $\mathrm{C}$ with an initial voltage $\mathrm{V}_{0}$ at $\mathrm{t}=0$. Fig. 2 shows the magnetic flux density distribution of the conventional TC design at $\mathrm{t}=2 \mathrm{~ms}$. In the figure distortion of the flux lines is observed above the coil, where the armature is located. However below the coil the flux lines are unaltered, suggesting that interaction with a second armature may be used to improve actuator operating efficiency.

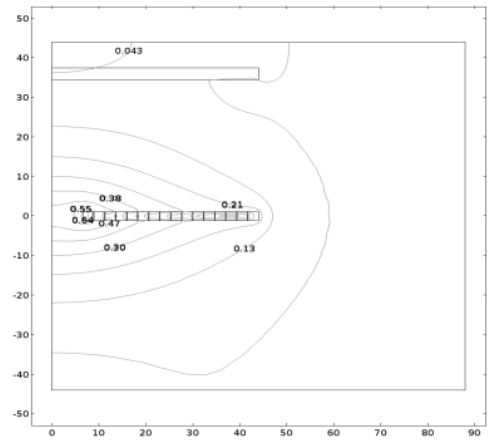

Fig. 2 TC actuator magnetic flux density norm (T) at $\mathrm{t}=2 \mathrm{~ms}$.

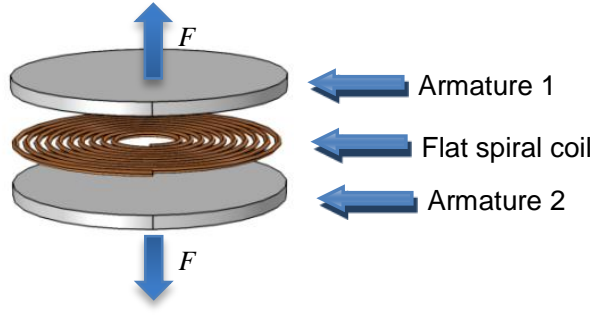

Fig. 3 DS-TC actuator schematic diagram.

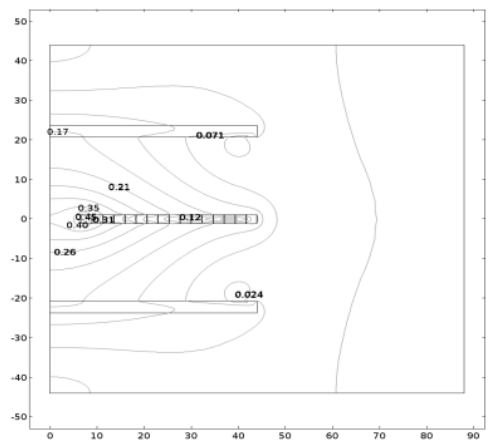

Fig. 4 DS-TC actuator magnetic flux density norm (T) at $\mathrm{t}=2$ ms.

Fig. 3 shows the proposed DS-TC arrangement with Fig. 4 showing the flux distribution obtained using the FE analysis. As can be observed in the simulation results, interaction with both armatures results in a symmetrical flux distribution, suggesting that performance gains by a DS-TC compared with a conventional TC actuator are possible.

\subsection{DS-TC actuator performance analysis}

For a kinetic energy $E$ and an armature mass $m$ the separation velocity of the single-sided TC armature is given by

$$
v=\sqrt{\frac{2 E}{m}}
$$

If radial symmetry along the flat spiral coil is assumed, it is not difficult to demonstrate that for the same kinetic energy and armature mass the separation velocity between armatures in a DS-TC is given by [14]:

$$
v=2 \sqrt{\frac{E}{m}}
$$

Thus for identical kinetic energies, the separation velocity between armatures is $\sqrt{2}$ higher for a DS-TC than the single armature velocity for a conventional TC design. This suggests that a DS-TC actuator is better suited for ultra-high speed applications than a TC design, providing the kinetic energy of both armatures can be effectively used. Although this analysis gives an insight into one advantage of the DS-TC over the single-sided design, it does not offer any time-line about the energy conversion process. Time quantification is essential in ultra-high speed applications, to fill this information void FE simulations were conducted. In these simulations, the 
electrical excitation source and the moving mass attached to the armature are made identical to ensure a fair comparison. At a time when the kinetic energy of both actuators is identical, the performance indicators of the two designs are compared. The TC and DS-TC parameters used in the simulations are listed in Table 1. Additionally, low value resistors and inductors $(9.1 \mathrm{~m} \Omega$ and $3.6 \mu \mathrm{H}$, respectively) were connected in series with the idealized coil to emulate the effect of connection leads. Fig. 5 and Fig. 6 show simulation results of velocity, displacement and kinetic energy for the investigated TC and DS-TC designs, respectively.

From the simulations it can be seen that a kinetic energy of $100 \mathrm{~J}$ is reached at $0.67 \mathrm{~ms}$ for the TC and at $1.14 \mathrm{~ms}$ for the DS-TC actuators. At these instants the armature velocities are $17.4 \mathrm{~m} / \mathrm{s}$ and $24.6 \mathrm{~m} / \mathrm{s}$ for the TC and DS-TC, respectively. The velocities are in-line with the expected values based in (1)-(2), validating the theoretical analysis and numerical simulation in the process. However, the $100 \mathrm{~J}$ of kinetic energy was reached considerably sooner by the TC than by the DS-TC, hence showing the better use of injected electrical energy (energy conversion efficiency) by the TC actuator. Furthermore, at the end of the $2 \mathrm{~ms}$ simulation the armature displacement of the TC actuator and the separation between armatures of the DS-TC are very similar; making the DS-TC unattractive due to its more complex construction and associated costs. These results clearly illustrate that for similarly sized devices the DS-TC is not automatically a better alternative to the conventional TC design, since depending on actuator parameter and excitation values, a TC actuator may exhibit a higher efficiency than a DS-TC design. To conclusively prove that the DS-TC actuator is better than a conventional TC design, superior energy conversion efficiency needs to be demonstrated. Refinement of the DS$\mathrm{TC}$ actuator is therefore a requirement.

\section{DS-TC actuator sensitivity analysis}

To better understand how the different design parameters affect the mechanical performance of a DS-TC actuator a sensitivity analysis of the device was conducted using FEA software. In the analysis a single actuator parameter was varied at a time, while the others parameters were kept at their starting values. The actuator starting values are listed in Table 1. Fig. 7 shows simulations of armature displacement against variations in actuator parameters.

\begin{tabular}{|l|l|l|}
\hline Parameter & Value & Description \\
\hline $\mathrm{V0}$ & $300 \mathrm{~V}$ & Capacitor voltage \\
\hline $\mathrm{C}$ & $30 \mathrm{mF}$ & Capacitance \\
\hline $\mathrm{Cn}$ & 16 & Number of turns \\
\hline $\mathrm{At}$ & $3 \mathrm{~mm}$ & Armature thickness \\
\hline $\mathrm{Ar}$ & $44 \mathrm{~mm}$ & Armature radius \\
\hline $\mathrm{Ch}$ & $2.24 \mathrm{~mm}$ & Conductor height \\
\hline $\mathrm{Cw}$ & $2.24 \mathrm{~mm}$ & Conductor width \\
\hline
\end{tabular}

Table 1: TC and DS-TC parameters
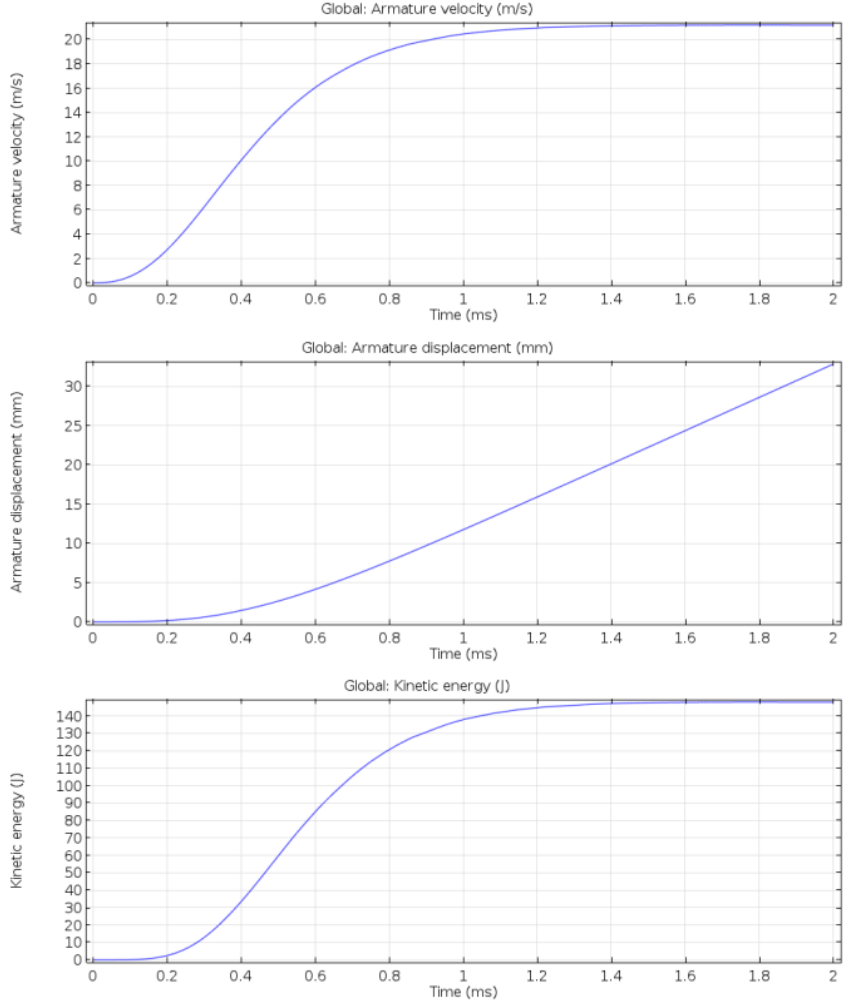

Fig. 5 TC actuator FE simulation results.
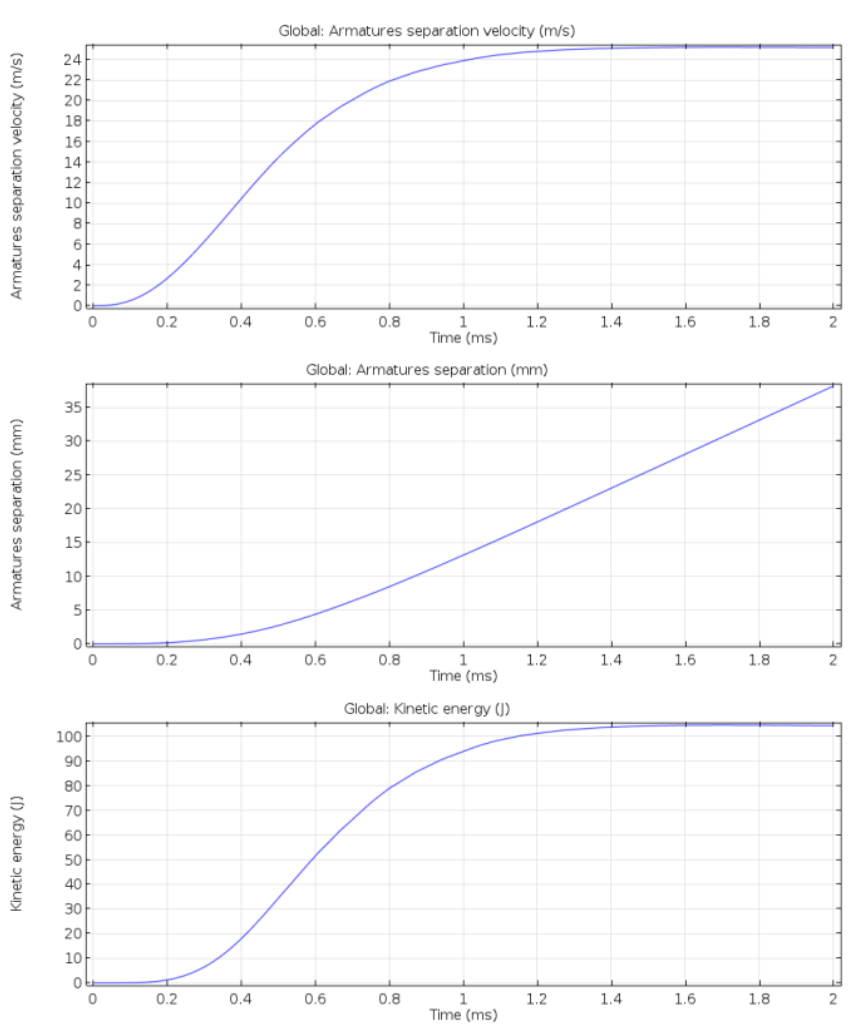

Fig. 6 DS-TC actuator FE simulation results. 


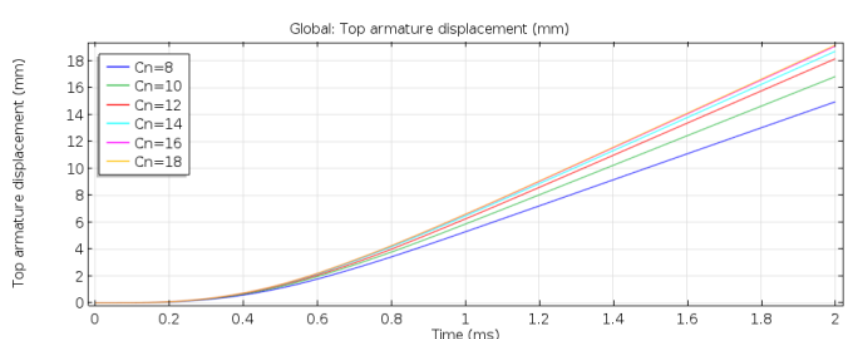

Global: Top armature displacement (mm)

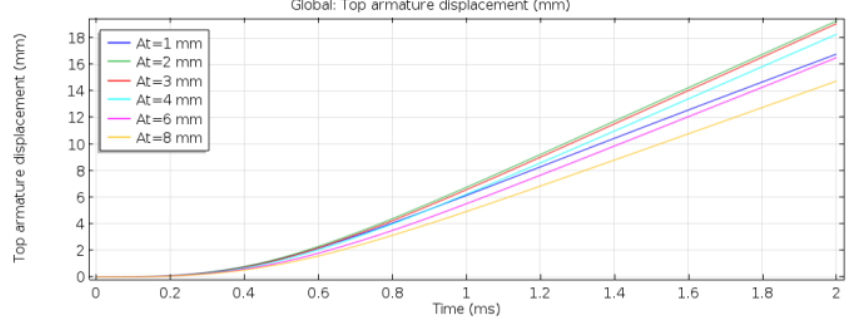

Global: Top armature displacement ( $\mathrm{mm}$ )
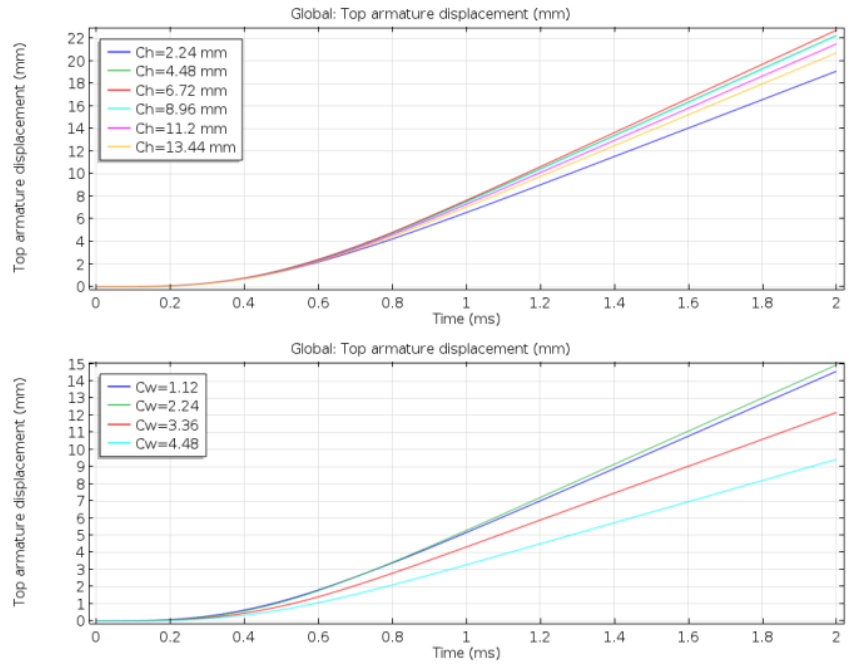

Fig. 7 DS-TC displacement for different parameter variation.

Based on the simulations a series of rules governing the behaviour of the DS-TC can be established:

- Increasing the input electrical energy results in higher, armature displacement, speed and actuator efficiency values. However the manner in which the electrical energy is shared between capacitor voltage and capacitance is of primary importance for the efficiency of the DS-TC. An optimal voltagecapacitance combination exists.

- Increasing the number of coil turns increases the armature speed, displacement, velocity, force and actuator efficiency.

- Increasing the armature thickness improves armature displacement, speed and actuator efficiency until a critical thickness is reached, after which the actuator performance degrades.

- An increase in conductor height is beneficial for armature displacement, speed, force and actuator efficiency until a critical height is reached, after which further increase is detrimental to actuator performance.
- Moving mass minimization is critical for actuator mechanical performance

The set of rules listed above does not differ drastically from optimization criteria published for the TC actuator [15], illustrating the similar operating principle of both devices.

\subsection{DS-TC actuator performance assessment}

Based on the set of rules above and using an iterative approach, the DS-TC actuator design was refined. The performance of the DS-TC actuator was then compared with that of a TC actuator. To enable a fair comparison, a similar optimisation procedure was also used to improve the performance of the TC design. During the FE simulations a total mechanical load of $1 \mathrm{~kg}$, evenly distributed over the total number of armatures, is assumed in both actuators, in order to have similar total moving mass in both systems; a $0.5 \mathrm{~kg}$ load on each armature for the DS-TC and a $1 \mathrm{~kg}$ load over the single armature of the TC actuator. Fig. 8 shows half the geometry of the resulting optimized DS-TC design.

Given that the voltage-capacitance excitation combination is known to have a significant impact on the performance of magnetic repulsion based actuators [13], a number of combinations were tried in order to identify the best suited for the two actuator designs under analysis. The combinations considered in this analysis are identical to those used in [13] to enable a straight forward comparison with published results. Table 2 and Table 3 summarize the actuator parameters and performance indicators obtained with the most effective voltage-capacitance combination identified from a $2 \mathrm{~ms}$ FE simulation for the DS-TC and TC actuators, respectively. The reported percentage of electrical to mechanical energy conversion efficiency was calculated as the ratio of kinetic energy achieved to electrical energy consumed, multiplied by 100 . To allow a simpler comparison between designs the same voltage-capacitance combination is used in both tables. It should be noted however, that for the DS-TC actuator a $727 \mathrm{~V}-10 \mathrm{mF}$ combination produced very similar, but slightly lower, efficiency and displacement values.

For the optimized single-sided TC actuator an efficiency of $29 \%$ was obtained from the simulations, while $34 \%$ was calculated for the DS-TC design, as shown in Tables 2 and 3. As a reference a much lower $14 \%$ efficiency was reported in [13] for a single-sided TC operating under similar conditions. The results in Table 2 clearly illustrate that a DS-TC is capable of operation at a higher efficiency than a single-sided TC design. It is important to mention that the displacement value reported in Table 2 for the DS-TC corresponds to that of a single armature. If the separation between armatures is considered instead, the total relative displacement doubles to $130 \mathrm{~mm}$; this observation is also valid for the relative peak force $(316 \mathrm{kN})$ and differential peak velocity value $(70 \mathrm{~m} / \mathrm{s})$. Here is where the advantage of the highly efficient DS-TC design resides: for the same time-lapse the separation between armatures of the DS-TC is about double the armature 
displacement of the TC actuator. Thus the DS-TC actuator is better suited for ultra-fast switch operation, if symmetrical electrode separation is allowed. In that case, mechanisms can be used to convert the opposing symmetrical displacement of the DS-TC armatures to a single side displacement [16], exploiting in this case the higher force produced by the device. Based on the above analysis and the reported simulation results the performance of a highly efficient DS TC is far superior to that of its single-sided counterpart. However careful fine tuning of the DS-TC is required.

\section{Conclusions}

The factors that affect the efficiency of the DS-TC actuator were investigated in this paper by means of a sensitivity analysis using FEA software. Based on a series of rules established as a consequence of the sensitivity assessment results, an iterative approach was used to improve the performance of a DS-TC design, resulting in a highly efficient actuator. The new design was shown to achieve a higher efficiency than those reported in the open literature for magnetic repulsion based actuators under similar operating conditions, even surpassing a highly efficient TC actuator developed during this research work. Table 4 summarizes the energy conversion efficiency for the actuator designs considered in the comparisons. It is believed that the double armature arrangement allows for a better use of the coil produced magnetic flux, resulting in a higher efficiency of the DS-TC actuator compared with that achievable using the conventional single-sided design. The higher efficiency of the implemented DS-TC actuator is reflected as a superior mechanical performance, exceeding substantially the capabilities of conventional TC designs.

A highly efficient DS-TC actuator has the potential not only to surpass the performance of existing magnetic repulsion based designs, but also to enable innovative mechanical solutions for ultra-fast switch operation.

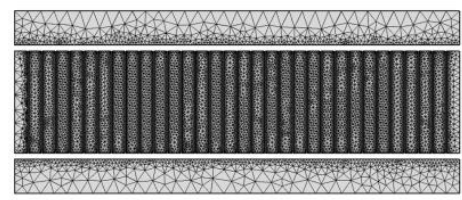

Fig. 8 DSTC half axial symmetry geometry.

\begin{tabular}{|l|l|l|l|}
\hline Parameter & Value & Parameter & Value \\
\hline Turns & 32 & Displacement $(\mathrm{mm})$ & 65 \\
\hline V0 $(\mathrm{V})$ & 2298 & Efficiency $(\%)$ & 34 \\
\hline C $(\mathrm{mF})$ & 1 & Peak Force $(\mathrm{kN})$ & 158 \\
\hline Armature Thickness $(\mathrm{mm})$ & 3 & Peak current $(\mathrm{kA})$ & 17 \\
\hline Wire cross section $\left(\mathrm{mm}^{2}\right)$ & $1.12 \times 4.48$ & Peak velocity $(\mathrm{m} / \mathrm{s})$ & 35 \\
\hline
\end{tabular}

Table 2: DS-TC actuator simulation results.

\begin{tabular}{|l|l|l|l|}
\hline Parameter & Value & Parameter & Value \\
\hline Turns & 32 & Displacement $(\mathrm{mm})$ & 69 \\
\hline V0 $(\mathrm{V})$ & 2298 & Efficiency $(\%)$ & 29 \\
\hline C $(\mathrm{mF})$ & 1 & Peak Force $(\mathrm{kN})$ & 257 \\
\hline Armature Thickness $(\mathrm{mm})$ & 3 & Peak current $(\mathrm{kA})$ & 13 \\
\hline Wire cross section $\left(\mathrm{mm}^{2}\right)$ & $1.12 \times 3.36$ & Peak velocity $(\mathrm{m} / \mathrm{s})$ & 36 \\
\hline
\end{tabular}

Table 3: TC actuator simulation results.

\begin{tabular}{|c|c|c|c|}
\hline & TC in [13] & TC & DS-TC \\
\hline Eff. (\%) & 14 & 29 & 34 \\
\hline
\end{tabular}

Table 4: Actuators electrical to mechanical energy conversion efficiency in percentage.

\section{Acknowledgements}

This work was funded as part of the UK EPSRC, FCL/B: An Integrated VSC-HVDC Fault Current Limiter/Breaker project, EP/L021552/1.

\section{References}

[1] J.W. Feltes, B.D. Gemmell, D. Retzmann, "From Smart Grid to Super Grid: Solutions with HVDC and FACTS for grid access of renewable energy sources," Power and Energy Society General Meeting, 2011 IEEE , vol., no., pp.1,6, 24-29 July 2011

[2] D. Van Hertem, M. Ghandhari, Multi-terminal VSC HVDC for the European supergrid: Obstacles, Renewable and Sustainable Energy Reviews, Volume 14, Issue 9, December 2010, Pages 3156-3163.

[3] T.K. Vrana, R.E. Torres-Olguin, B. Liu, T.M. Haileselassie, "The North Sea Super Grid - a technical perspective," AC and DC Power Transmission, 2010. ACDC 9th IET International Conference on, vol., no., pp.1,5, 19-21 Oct. 2010

[4] N. Flourentzou, V. Agelidis, and G. Demetriades, "VSCBased HVDC Power Transmission Systems: An Overview,"

[5] C.M. Franck, "HVDC Circuit Breakers: A Review Identifying Future Research Needs," Power Delivery, IEEE Transactions on, vol.26, no.2, pp.998,1007, April 2011

[6] J. Häfner, B. Jacobson, "Proactive Hybrid HVDC Breakers - A key innovation for reliable HVDC grids," (Cigré Bologna, Paper 0264, 2011)

[7] C. Meyer and R. De Doncker, "Solid-state circuit breaker based on active thyristor topologies," IEEE Trans. Power Electron., vol. 21, pp. 450-458, Mar. 2006.

[8] H. Pang, G. Tang, and Z. He, "Evaluation of losses in VSC-HVDC transmission system," in IEEE Power and Energy Society General Meeting - Conversion and Delivery of Electrical Energy in the 21st Century, Pittsburgh, PA, USA, Jul. 2008, pp. $1-6$. 
[9] W. Holaus, K. Frohlich, "Ultra-fast switches- a new element for medium voltage fault current limiting switchgear," Power Engineering Society Winter Meeting, 2002. IEEE, vol.1, no., pp.299,304 vol.1, 2002

[10] M. Steurer, K. Frohlich, W. Holaus, and K. Kaltenegger, "A novel hybrid current-limiting circuit breaker for medium voltage: Principle and test results," IEEE Trans. Power Del., vol. 18, no. 2, pp. 460-467, Apr. 2003.

[11] A. Bissal, J. Magnusson, G. Engdahl, "Comparison of Two Ultra-Fast Actuator Concepts," Magnetics, IEEE Transactions on , vol.48, no.11, pp.3315,3318, Nov. 2012

[12] V. Puumala, L. Kettunen, "Electromagnetic Design of Ultrafast Electromechanical Switches," Power Delivery, IEEE Transactions on, 2014.

[13] A. Bissal, J. Magnusson, G. Engdahl, "Electric to Mechanical Energy Conversion of Linear Ultrafast Electromechanical Actuators Based on Stroke Requirements," Industry Applications, IEEE Transactions on , vol.51, no.4, pp.3059,3067, July-Aug. 2015

[14] J. Ostrowski, "Electrical switch with Thomson coil drive", WO 2014048483 A1, April 3, 2014.

[15] D. Enyuan, W. Yongxing, C. Jiyuan, Z. Jiyan, "The analysis of high-speed repulsion actuator and performance comparisons with permanent magnetic actuator in vacuum circuit breaker," Discharges and Electrical Insulation in Vacuum, 2008. ISDEIV 2008. 23rd International Symposium on , vol.1, no., pp.189-191, 15-19 Sept. 2008.

[16] N. Sclater, and N.P. Chironis, "Mechanisms and mechanical devices sourcebook," 4th edition, New York: McGraw-Hill, 2007. 